\title{
Quantitative weighted mixed weak-type inequalities for classical operators
}

\author{
S. Ombrosi, C. Pérez and J. Recchi
}

Indiana J. of Math. (to appear)

\begin{abstract}
We improve on several mixed weak type inequalities both for the Hardy-Littlewood maximal function and for Calderón-Zygmund operators. These type of inequalities were considered by Muckenhoupt and Wheeden and later on by Sawyer estimating the $L^{1, \infty}(u v)$ norm of $v^{-1} T(f v)$ for special cases. The emphasis is made in proving new and more precise quantitative estimates involving the $A_{p}$ or $A_{\infty}$ constants of the weights involved.
\end{abstract}

\section{Introduction and statements of the main results}

Let $M$ denote the usual Hardy-Littlewood maximal function, then according to a fundamental result of $\mathrm{B}$. Muckenhoupt $[\mathrm{Mu}], M$ is a bounded operator on the Lebesgue space $L^{p}(d \mu), 1<p<+\infty$, if and only if $d \mu=w(x) d x$ and the weight $w$ satisfies the simple geometric condition

$$
[w]_{A_{p}}:=\sup _{Q}\left(\frac{1}{|Q|} \int_{Q} w\right)\left(\frac{1}{|Q|} \int_{Q} w^{1-p^{\prime}}\right)^{p-1}<\infty,
$$

where the supremum is taken over all cubes $Q$ in $\mathbb{R}^{n}$. This is the celebrated Muckenhoupt $A_{p}$ condition. A similar result holds in the case $p=1$, namely $M$ is of weak type $(1,1)$ with respect to $\mu$, i.e. $M: L^{1}(\mu) \rightarrow L^{1, \infty}(\mu)$, if and only if $d \mu=w(x) d x$ and the weight $w$ satisfies the $A_{1}$ condition,

$$
[w]_{A_{1}}:=\sup _{Q}\left(\frac{1}{|Q|} \int_{Q} w\right)\left(\underset{Q}{\operatorname{ess} \inf w)^{-1}<\infty}\right.
$$

where, again, the supremum is taken over all cubes $Q$ in $\mathbb{R}^{n}$.

Since the $A_{p}$ theorem of Muckenhoupt plays a central role in modern Harmonic Analysis, different proofs from the original one in $[\mathrm{Mu}]$ have been considered in the literature.

2010 Mathematics Subject Classification: 42B20, 42B25, 46E30.

Key words and phrases: maximal operators, Calderón-Zygmund operators, weighted estimates.

The first and third authors are supported by Universidad Nacional del Sur and CONICET.

The second author was supported by grant MTM2014-53850-P, Spanish Government 
In particular, E. Sawyer tried in $[\mathrm{Sa}$ ] the following approach based on the factorization theorem for $A_{p}$ weights of P. Jones (see $[\mathrm{GCRdF}]$ ). Recall that a weight $w$ satisfies the $A_{p}$ condition if and only if there are two $A_{1}$ weights $u$ and $v$ such that

$$
w=u v^{1-p} .
$$

Then, if the following operator is defined

$$
S f=\frac{M(v f)}{v}
$$

the boundedness of $M$ on $L^{p}(w)$ may be rewritten as

$$
\int_{\mathbb{R}^{n}}|S f|^{p} u v d x \leq c \int_{\mathbb{R}^{n}}|f|^{p} u v d x .
$$

Observe now that since $v \in A_{1}, M v \leq[v]_{A_{1}} v$ and hence $S$ is bounded in $L^{\infty}(u v)$. Therefore, if we show that $S$ is of weak type $(1,1)$ with respect to the measure $u v d x$ we can apply the Marcinkiewicz interpolation theorem to derive (2). This is precisely the statement of the following theorem from [Sa].

Theorem 1.1 If $u, v \in A_{1}(\mathbb{R})$, then

$$
\left\|\frac{M(g)}{v}\right\|_{L^{1, \infty}(u v)} \leq c\|g\|_{L^{1}(u)},
$$

where $c$ depends only on the $A_{1}$ constant of $u$ and the $A_{1}$ constant of $v$. This shows that the operator $S f=v^{-1} M(v f)$ is of weak type $(1,1)$ with respect to the measure vudx.

In the same article, Sawyer conjectured that this theorem should also hold for the maximal function in $\mathbb{R}^{n}$ and for the the Hilbert transform $H$ instead of $M$.

The article of Sawyer was also very much motivated by a previous work of B. Muckenhoupt and R. Wheeden [MW]. The main result of this paper holds this time for both the one dimensional Hardy-Littlewood maximal function and the Hilbert transform. To be more precise, the main result proved in [MW] is the following.

Theorem 1.2 Let $w \in A_{1}(\mathbb{R})$, there exists a constant $c$ such that,

$$
\left\|M\left(f w^{-1}\right) w\right\|_{L^{1, \infty}(\mathbb{R})} \leq c\|f\|_{L^{1}(\mathbb{R})}
$$

and

$$
\left\|H\left(f w^{-1}\right) w\right\|_{L^{1, \infty}(\mathbb{R})} \leq c\|f\|_{L^{1}(\mathbb{R})} .
$$

In [C-UMP1] the authors extended both Theorems 1.1 and 1.2 to $\mathbb{R}^{n}$ containing in particular the conjectures formulated by Sawyer mentioned above. The precise result is the following. 
Theorem 1.3 Suppose that $u \in A_{1}$ and that either $v \in A_{1}$ or $v \in A_{\infty}(u)$, then there exists a constant $c$ such that,

$$
\left\|\frac{M(f v)}{v}\right\|_{L^{1, \infty}(u v)} \leq c\|f\|_{L^{1}(u v)}
$$

and

$$
\left\|\frac{T(f v)}{v}\right\|_{L^{1, \infty}(u v)} \leq c\|f\|_{L^{1}(u v)},
$$

where $M$ is the Hardy-Littlewood maximal operator and $T$ is a Calderón-Zygmund operator.

We remark that this result holds for $T^{*}$, the maximal singular integral operator, instead of $T$. Given weights $u$ and $v$, by $v \in A_{\infty}(u)$ we mean that $v$ satisfies the $A_{\infty}$ condition defined with respect to the measure $u d x$ (as opposed to Lebesgue measure). A more precise definition is given in Section 2 below.

We emphasize that this theorem contains both Theorems 1.1 and 1.2 as particular cases. Indeed, the case of the first theorem is clear. For the second, if $w \in A_{1}$, we let $u=w$ and $v=w^{-1}$. Then, $u v=1 \in A_{\infty}$ and thus $v \in A_{\infty}(u)$ by Lemma 2.1 and Observation 2.2.

To prove Theorem 1.3, the authors show that it suffices to prove the result for the dyadic maximal function $M_{d}$ by proving an extrapolation type theorem, Theorem 1.5 below, that allows to replace $T$ or $M$ by $M_{d}$. To be more precise, the combination of the following two theorems from [C-UMP1] proves Theorem 1.3.

Theorem 1.4 Suppose that $u \in A_{1}$ and that either $v \in A_{1}$ or $v \in A_{\infty}(u)$, then there exists a constant $c$ such that,

$$
\left\|\frac{M_{d}(f v)}{v}\right\|_{L^{1, \infty}(u v)} \leq c\|f\|_{L^{1}(u v)} .
$$

Theorem 1.5 Given a family $\mathcal{F}$ of pair of functions, suppose that for some $p \in(0, \infty)$ and for every $w \in A_{\infty}$,

$$
\|f\|_{L^{p}(w)} \leq C\|g\|_{L^{p}(w)},
$$

for all $(f, g) \in \mathcal{F}$ such that the left-hand side is finite, and where $C$ depends only on the $A_{\infty}$ constant of $w$. Then for all weights $u \in A_{1}$ and $v \in A_{\infty}$,

$$
\left\|f v^{-1}\right\|_{L^{1, \infty}(u v)} \leq C\left\|g v^{-1}\right\|_{L^{1, \infty}(u v)} \quad(f, g) \in \mathcal{F} .
$$

Here $\mathcal{F}$ denotes a family of ordered pairs of non-negative, measurable functions $(f, g)$.

Theorem 1.5 from [C-UMP1] is used to pass from $M$ to $M_{d}$ since by standard methods, for every $p \in(0, \infty)$ and every $w \in A_{\infty}$

$$
\|M(f v)\|_{L^{p}(w)} \leq c\left\|M_{d}(f v)\right\|_{L^{p}(w)},
$$


where the constant $c$ involves the $A_{\infty}$ constant of $w$. However, there are recent results showing that Theorem 1.5 can be avoided in the transition from $M$ to $M_{d}$. Indeed, using for instance $[\mathrm{HP}]$ p. 792 , we have that

$$
M f \leq c_{n} \sum_{\alpha \in\left\{0, \frac{1}{3}\right\}^{n}} M_{d}^{\alpha} f .
$$

where $M_{d}^{\alpha}$ is an appropriate shifted dyadic maximal function with similar properties as $M_{d}$. Thus, the expression on the left in (5) is bounded by a dimensional constant multiple of the corresponding expression for $M_{d}^{\alpha}$. Since each of these $M_{d}^{\alpha}$ has similar properties as $M_{d}$ the corresponding proof of (7) is exactly the same.

In [C-UMP1] the authors conjectured that Theorem 1.4 still holds under milder hypotheses on the weight $v$. To be more precise, the authors state what is now known as "Sawyer's Conjecture", although E. Sawyer never asserted it. The conjecture is the following.

Conjecture 1.6 Suppose that $u \in A_{1}$ and $v \in A_{\infty}$. Then there exists a constant c such that

$$
\left\|\frac{M_{d}(f v)}{v}\right\|_{L^{1, \infty}(u v)} \leq c\|f\|_{L^{1}(u v)} .
$$

Note that if $v \in A_{\infty}(u)$ (always assuming $u \in A_{1}$ ), then $v \in A_{\infty}$ (see Lemmas 2.1 and 2.3). This conjecture has been open for several years and has been studied by different authors.

In this paper we try to understand the difficulties of this conjecture and propose alternative ways to prove it. We will also study how the constants of the weights $u$ and $v$ is reflected in these inequalities, that is, we look for quantitative versions of this type of inequalities.

The first question that we pose concerning Sawyer's Theorem is the following:

What is the sharp dependence on the constants of the weights $u$ and $v$ when both are in $A_{1}$ ?

Following the proof given in [C-UMP1], which is an adaptation of the original proof given by Sawyer in [Sa] for the real line, we show the dependence on the weight constants. More specifically, we prove the following result.

Theorem 1.7 If $u \in A_{1}$ and $v \in A_{1}$, there exists a dimensional constant $c$ such that

$$
\left\|\frac{M_{d}(f v)}{v}\right\|_{L^{1, \infty}(u v)} \leq c[u]_{A_{1}}^{2}[v]_{A_{1}}^{4}\|f\|_{L^{1}(u v)} .
$$

The proof may be found in Section 7 .

We believe that the dependency on the constants in inequality (9) is not sharp since the method does not seem to be adequate. Trying to understand this issue we will focus on the special case $u=1$ which is interesting in its own. The finiteness of the estimate in this special case is assured by Theorem 1.3 assuming even a weaker condition on $v$ 
than $A_{1}$, namely $v \in A_{\infty}(u)=A_{\infty}$. The method that we use is different from the one considered in the proof of Theorem 1.7 allowing us to obtain more precise estimates. In particular we will prove the linearity of the constant bound of the weight $v$ if we assume the stronger condition $v \in A_{1}$ and the result is sharp. Our theorem is the following.

Theorem 1.8 Let $v \in A_{1}$. There exists a dimensional constant $c$, independent from $[v]_{A_{1}}$, such that

$$
\left\|\frac{M(f)}{v}\right\|_{L^{1, \infty}(v)} \leq c[v]_{A_{1}}\|f\|_{L^{1}\left(\mathbb{R}^{n}\right)} .
$$

Furthermore, the linear dependence on $[v]_{A_{1}}$ is sharp.

However, we want to understand the more general case.

Problem 1.9 Find an increasing function $\varphi:[1, \infty] \rightarrow[1, \infty]$ for which the following inequality holds whenever $v \in A_{\infty}$

$$
\left\|\frac{M(f)}{v}\right\|_{L^{1, \infty}(v)} \leq c \varphi\left([v]_{A_{\infty}}\right)\|f\|_{L^{1}\left(\mathbb{R}^{n}\right)},
$$

where $c$ is a constant that depends on the dimension.

This problem is a special case of Conjecture 1.6 with $u=1$ and it will be studied In Section 4. The best constant in (11), $\varphi\left([v]_{A_{\infty}}\right)$, is finite by Theorem 1.3. Our goal is to determine the best dependence on the constant of the weight $v$ or, in other words, to find the smallest function $\varphi$. Recall that $A_{\infty}=\cup_{p \geq 1} A_{p}$ and that, if $w \in A_{\infty}$ we use the weight constant

$$
[w]_{A_{\infty}}:=\sup _{Q} \frac{1}{w(Q)} \int_{Q} M\left(\chi_{Q} w\right) d x
$$

called the Fujii-Wilson constant in some recent papers. We could use instead the constant defined by Hrushev in $[\mathrm{Hr}]$ which is more natural, however it was shown in [HP] that it is much larger than the one given by the functional (12).

We remark here that a condition on the weight $v$ in (10) or (11) must be taken into account. Indeed, there are estimates like

$$
\left\|\frac{M(f)}{M w}\right\|_{L^{1, \infty}(M w)} \leq c\|f\|_{L^{1}\left(\mathbb{R}^{n}\right)},
$$

namely with $v=M w$, which are false for a general function $w$ or measure. This will be shown in Section 5 where, furthermore, an interesting relationship with the two weight problem for singular integrals is implicit in the argument. In general, weights of the form $M w$ are not $A_{\infty}$ weights but small perturbations, namely when $v=(M w)^{\delta}, \delta \in(0,1)$, makes the inequality to be true since in this case $v \in A_{1}$ and Theorem 10 applies. It is interesting that in special situations and for large perturbations of the weight the result is still true. Indeed, if $v(x)=|x|^{-n r} \approx(M \delta)^{r}$ with $r>1$, then there is a finite constant $c$ such that

$$
\left\|\frac{M(f)}{v}\right\|_{L^{1, \infty}(v)} \leq c\|f\|_{L^{1}\left(\mathbb{R}^{n}\right)}
$$


being the result false in the case $r=1$. This was proved in dimension one by Andersen and Muckenhoupt in $[\mathrm{AM}]$ and by Martín-Reyes, Ortega Salvador and Sarrión Gavián [MOS] in higher dimensions. We remark that these weights $v(x)=|x|^{-n r}$ are not $A_{\infty}$ weights.

In view of Theorem 1.8 and the case $v=1$ we state the following conjecture for the general case.

Conjecture 1.10 Let $u \in A_{1}$ and $v \in A_{1}$, then there exists a dimensional constant $c$ such that

$$
\left\|\frac{M_{d}(f v)}{v}\right\|_{L^{1, \infty}(u v)} \leq c[u]_{A_{1}}[v]_{A_{1}}\|f\|_{L^{1}(u v)} .
$$

To see that the dependency cannot be better than $[u]_{A_{1}}[v]_{A_{1}}$ we prove the following result which strengthens our conjecture.

Theorem 1.11 Let $u \in A_{1}, v \in A_{1}$. If

$$
\left\|\frac{M_{d}(f v)}{v}\right\|_{L^{1, \infty}(u v)} \leq c \varphi\left([u]_{A_{1}},[v]_{A_{1}}\right)\|f\|_{L^{1}(u v)},
$$

then, there is a constant $c$ independent of the weights such that

$$
\varphi\left([u]_{A_{1}},[v]_{A_{1}}\right) \geq c[u]_{A_{1}}[v]_{A_{1}} .
$$

Another related problem, partly intermediate between the previous two problems, would be to determine how the dependence on the constant $[v]_{A_{p}}$ is if we assume that $v \in A_{p}$ for some $p \geq 1$. We should also take into account that Theorem 1.8 gives the sharp dependence on the real line when assuming the stronger assumption $v \in A_{1}$. Based on this we state the following conjecture.

Conjecture 1.12 Let $v \in A_{p}, p \geq 1$, then there exists a dimensional constant $c$ such that

$$
\left\|\frac{M_{d}(f v)}{v}\right\|_{L^{1, \infty}(v)} \leq c[v]_{A_{p}}\|f\|_{L^{1}(v)} .
$$

We were not able to prove this conjecture but we have obtained the following result using an adequate Calderón-Zygmund decomposition that involves the $A_{\infty}$ constant of the weight.

Theorem 1.13 Let $v \in A_{p}, p \geq 1$, then there exists a dimensional constant $c$ such that

$$
\left\|\frac{M_{d}(f v)}{v}\right\|_{L^{1, \infty}(v)} \leq c[v]_{A_{\infty}} \max \left\{p, \log \left(e+[v]_{A_{p}}\right)\right\}\|f\|_{L^{1}(v)} .
$$

Corollary 1.14 Let $v \in A_{p}, p \geq 1$, then there exists a dimensional constant $c$ such that

$$
\left\|\frac{M_{d}(f v)}{v}\right\|_{L^{1, \infty}(v)} \leq C_{n}[v]_{A_{p}} \max \left\{p, \log \left(e+[v]_{A_{p}}\right)\right\}\|f\|_{L^{1}(v)} .
$$


We also try to improve the dependency on the weight constant using some other refined constants that were introduced in [HP] and formalized in the work of Lerner and Moen $[\mathrm{LM}]$.

Theorem 1.15 Let $v \in A_{p}, p \geq 1$, then there exists a dimensional constant $c$ such that

$$
\left\|\frac{M_{d}(f v)}{v}\right\|_{L^{1, \infty}(v)} \leq c p[v]_{\left(A_{p}\right)^{1 / p}\left(A_{\infty}^{e x p}\right)^{1 / p^{\prime}}} \log \left(e+[v]_{\left(A_{p}\right)^{1 / p}\left(A_{\infty}^{e x p}\right)^{1 / p^{\prime}}}\right)\|f\|_{L^{1}(v)} .
$$

We remit to Section 2 for the definition of $[v]_{\left(A_{p}\right)^{1 / p}\left(A_{\infty}^{e x p}\right)^{1 / p^{\prime}}}$.

In this paper we will also study similar problems for Calderón-Zygmund operators instead of the Hardy-Littlewood maximal function. In particular, we will improve the following theorem from $[\mathrm{HP}]$.

Theorem 1.16 Suppose that $T$ is a Calderón-Zygmund Operator, then there is a dimensional constant $c$ such that for any $v \in A_{1}$

$$
\left\|\frac{T(f v)}{v}\right\|_{L^{1, \infty}(v)} \leq c[v]_{A_{1}} \log \left(e+[v]_{A_{\infty}}\right)\|f\|_{L^{1}(v)} .
$$

This theorem improved the following result previously obtained in [LOP2].

$$
\left\|\frac{T(f v)}{v}\right\|_{L^{1, \infty}(v)} \leq c[v]_{A_{1}} \log \left(e+[v]_{A_{1}}\right)\|f\|_{L^{1}(v)} .
$$

In section 5, we will give a version of Corollary 1.14 for Calderón-Zygmund operators. We will prove the following result.

Theorem 1.17 Suppose that $T$ is a Calderón-Zygmund Operator, then there is a dimensional constant $c$ such that for any $v \in A_{p}$

$$
\left\|\frac{T(f v)}{v}\right\|_{L^{1, \infty}(v)} \leq c[v]_{A_{p}} \max \left\{p, \log \left(e+[v]_{A_{p}}\right)\right\}\|f\|_{L^{1}(v)} .
$$

\section{Preliminaries}

As usual a weight will be a nonnegative locally integrable function. Given a weight $w$, $p \in(1, \infty)$ and a cube $Q$ we denote

$$
A_{p}(w ; Q):=\left(\frac{1}{|Q|} \int_{Q} w\right)\left(\frac{1}{|Q|} \int_{Q} w^{1-p^{\prime}}\right)^{p-1}=\frac{w(Q) \sigma(Q)^{p-1}}{|Q|^{p}}
$$

where $\sigma=w^{-\frac{1}{p-1}}$. When $p=1$ we define the limiting quantity as

$$
A_{1}(w ; Q):=\left(\frac{1}{|Q|} \int_{Q} w\right)\left(\inf _{Q} w\right)^{-1}=\lim _{p \rightarrow 1} A_{p}(w, Q) .
$$


For $p=\infty$ we will consider two constants. The first constant is defined as a limit of the $A_{p}(w ; Q)$ constants

$$
A_{\infty}^{e x p}(w ; Q):=\left(\frac{1}{|Q|} \int_{Q} w\right) \exp \left(\frac{1}{|Q|} \int_{Q} \log w^{-1}\right)=\lim _{p \rightarrow \infty} A_{p}(w, Q) .
$$

To define the second constant we let

$$
A_{\infty}^{W}(w ; Q):=\frac{1}{w(Q)} \int_{Q} M\left(\chi_{Q} w\right)
$$

and define:

$$
\begin{aligned}
{[w]_{A_{p}} } & =\sup _{Q} A_{p}(w ; Q), \\
\|w\|_{A_{\infty}} & =\sup _{Q} A_{\infty}^{\exp }(w ; Q)
\end{aligned}
$$

and

$$
[w]_{A_{\infty}}=\sup _{Q} A_{\infty}^{W}(w ; Q)
$$

We write $w \in A_{p}$ if $[w]_{A_{p}}<\infty$ and $w \in A_{\infty}$ if $\|w\|_{A_{\infty}}<\infty$ or $[w]_{A_{\infty}}<\infty$. The constant $\|w\|_{A_{\infty}}$ was defined by Hruščev in $[\mathrm{Hr}]$. The constant $[w]_{A_{\infty}}$ was defined by Fujii in $[\mathrm{F}]$ and rediscovered by M. Wilson in [W1, W3], who also showed that both constants define the class $A_{\infty}$. In [HP], the authors proved the estimate

$$
[w]_{A_{\infty}} \leq c_{n}\|w\|_{A_{\infty}}
$$

and provided examples showing that $\|w\|_{A_{\infty}}$ can be exponentially larger than $[w]_{A_{\infty}}$.

We now define the mixed type constants. Given $1 \leq p<\infty$ and $\alpha, \beta \geq 0$, motivated by some results for the two weighted estimate for the maximal function in [HP], Lerner and Moen in $[\mathrm{LM}]$ defined the following mixed constants:

$$
[w]_{\left(A_{p}\right)^{\alpha}\left(A_{r}\right)^{\beta}}=\sup _{Q} A_{p}(w ; Q)^{\alpha} A_{r}(w ; Q)^{\beta}, \quad 1 \leq r<\infty,
$$

the exponential mixed constants:

$$
[w]_{\left(A_{p}\right)^{\alpha}\left(A_{\infty}^{e x p}\right)^{\beta}}=\sup _{Q} A_{p}(w ; Q)^{\alpha} A_{\infty}^{e x p}(w ; Q)^{\beta},
$$

and the Fujii-Wilson mixed constants:

$$
[w]_{\left(A_{p}\right)^{\alpha}\left(A_{\infty}^{W}\right)^{\beta}}=\sup _{Q} A_{p}(w ; Q)^{\alpha} A_{\infty}^{W}(w ; Q)^{\beta} .
$$

If $\alpha>0$, the class of weights that satisfy

$$
[w]_{\left(A_{p}\right)^{\alpha}\left(A_{\infty}^{W}\right)^{\beta}}<\infty
$$

is simply the class $A_{p}$, since

$$
\max \left([w]_{A_{p}}^{\alpha},[w]_{A_{\infty}}^{\beta}\right) \leq[w]_{\left(A_{p}\right)^{\alpha}\left(A_{\infty}^{W}\right)^{\beta}} \leq[w]_{A_{p}}^{\alpha+\beta} .
$$




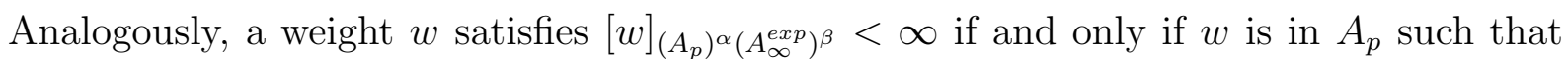
the inequality holds for the exponential mixed constant. In [LM] the author showed that if $0<\alpha \leq \beta \leq 1$ and $w \in A_{p}$, then

$$
[w]_{\left(A_{p}\right)^{\alpha}\left(A_{\infty}^{e x p}\right)^{1-\alpha}} \leq[w]_{\left(A_{p}\right)^{\beta}\left(A_{\infty}^{e x p}\right)^{1-\beta} .}
$$

We finish this section by defining the generalized $A_{\infty}$ class of weights $A_{\infty}(\mu)$ where $\mu$ is a doubling measure. To do this we recall some well known definitions about generalized Hardy-Littlewood maximal operators. For a complete account, we refer the reader to [D, GCRdF].

Given a doubling measure $\mu$ we define the maximal operator $M_{\mu}$ by

$$
M_{\mu} f(x)=\sup _{Q \ni x} \frac{1}{\mu(Q)} \int_{Q}|f(y)| d \mu(y) .
$$

For $1<p<\infty$, given a weight $w$ we say that $w \in A_{p}(\mu)$ if for all cubes $Q$,

$$
\left(\frac{1}{\mu(Q)} \int_{Q} w(x) d \mu(x)\right)\left(\frac{1}{\mu(Q)} \int_{Q} w(x)^{1-p^{\prime}} d \mu(x)\right)^{p-1} \leq C .
$$

We say that $w \in A_{1}(\mu)$ if

$$
M_{\mu} w(x) \leq C w(x) .
$$

We denote the union of all the $A_{p}(\mu)$ classes by $A_{\infty}(\mu)$, that is to say

$$
A_{\infty}(\mu)=\cup_{p \geq 1} A_{p}(\mu) .
$$

Since $\mu$ is doubling, then $M_{\mu}$ is bounded on $L^{p}(w d \mu), 1<p<\infty$, if and only if $w \in A_{p}(\mu)$. As usual when $\mu$ is the Lebesgue measure we omit the subscript $\mu$ and write simply $M$ or $A_{p}$. Also, if $\mu$ is absolutely continuous given by the weight $u$ then we simply write $A_{p}(u), 1 \leq p \leq \infty$.

The next two lemmas were proved in [C-UMP2].

Lemma 2.1 If $u \in A_{1}$ and $v \in A_{\infty}(u)$, then $u v \in A_{\infty}$. In particular, if $v \in A_{p}(u)$, $1 \leq p<\infty$, then $u v \in A_{p}$.

Observation 2.2 If $u \in A_{1}$, then $v \in A_{\infty}(u)$ if and only if $u v \in A_{\infty}$.

Lemma 2.3 If $u \in A_{1}$ and $u v \in A_{\infty}$, then $v \in A_{\infty}$.

\section{The $A_{1}$ case}

\section{Proof of Theorem 1.8.}

As usual we denote $M^{c}$ the centered Hardy-Littlewood maximal operators and its corresponding centered weighted $M_{v}^{c}$ maximal function. Now, by standard arguments

$$
\frac{M(f v)}{v} \approx \frac{M^{c}(f v)}{v} \leq \frac{M^{c} v}{v} M_{v}^{c}(f) \leq \frac{M v}{v} M_{v}^{c}(f) \leq[v]_{A_{1}} M_{v}^{c}(f)
$$


and then

$$
\begin{gathered}
\left\|\frac{M(f v)}{v}\right\|_{L^{1, \infty}(v)} \leq c_{n}[v]_{A_{1}}\left\|M_{v}^{c}(f)\right\|_{L^{1, \infty}(v)} \\
\leq c_{n}[v]_{A_{1}}\|f\|_{L^{1}(v)},
\end{gathered}
$$

by the Besicovitch covering lemma.

The proof will be completed by showing that the linear exponent is the best possible. To see this, it is sufficient to considerer $f(x)=\frac{1}{\delta} \chi_{(0,1)}(x)$ and $v(x)=|x|^{\delta-1}$ where $0<$ $\delta<1$. Then standard computations shows that

$$
[v]_{A_{1}} \sim \frac{1}{\delta}
$$

On the other hand, we can compute

$$
M(f v) \geq \begin{cases}\frac{1}{\delta} \frac{1}{x^{1-\delta}} & \text { if } x \in(0,1) \\ \frac{1}{\delta^{2}} \frac{1}{x} & \text { if } x \in(1, \infty) \\ \frac{1}{\delta^{2}} \frac{1}{1-x} & \text { if } x \in(-\infty, 0)\end{cases}
$$

therefore $\left(0, \delta^{-2 / \delta}\right) \subset\{x: M(f v)>v\}$. Continuing we have

$$
v\{x: M(f v)>v\} \geqslant v\left(0, \delta^{-2 / \delta}\right)=\int_{0}^{\delta^{-2 / \delta}} x^{\delta-1} d x=\frac{1}{\delta^{3}}=[v]_{A_{1}} \frac{1}{\delta^{2}},
$$

but $\int_{\mathbb{R}} f(x) v(x) d x=\int_{0}^{1} \frac{1}{\delta} x^{\delta-1} d x=\frac{1}{\delta^{2}}$.

Proof of Theorem 1.11. Let $f(x)=\frac{1}{\delta} \chi_{(0,1)}(x)$ and define $u(x)=\alpha \chi_{(0,1)}(x)+\chi_{(0,1)^{c}}(x)$, where $0<\alpha<1$ and $v(x)=|x|^{\delta-1}$, where $0<\delta<1$. Then standard computations shows that

$$
[u]_{A_{1}} \sim \frac{1}{\alpha} \quad \text { and } \quad[v]_{A_{1}} \sim \frac{1}{\delta}
$$

Also, we have

$$
M(f v) \geq \begin{cases}\frac{1}{\delta} \frac{1}{x^{1-\delta}} & \text { if } x \in(0,1) \\ \frac{1}{\delta^{2}} \frac{1}{x} & \text { if } x \in(1, \infty) \\ \frac{1}{\delta^{2}} \frac{1}{1-x} & \text { if } x \in(-\infty, 0) .\end{cases}
$$

Then, $\left(0, \delta^{-2 / \delta}\right) \subset\{x: M(f v)>v\}$ and then

$$
u v\{x: M(f v)>v\} \geqslant u v\left(1, \delta^{-2 / \delta}\right)=\int_{1}^{\delta^{-2 / \delta}} x^{\delta-1} d x=\frac{1}{\delta}\left(\delta^{-2}-1\right) \approx \frac{1}{\delta^{3}} .
$$

On the other hand,

$$
\int_{\mathbb{R}} f(x) u(x) v(x) d x=\frac{\alpha}{\delta} \int_{0}^{1} x^{\delta-1} d x=\frac{\alpha}{\delta^{2}},
$$


this proves $\varphi\left([u]_{A_{1}},[v]_{A_{1}}\right) \gtrsim[u]_{A_{1}}[v]_{A_{1}}$.

Observation 3.1 When considering the case $\alpha=\delta$, we have that $\varphi\left([u]_{A_{1}},[v]_{A_{1}}\right)$ cannot be $\max \left([u]_{A_{1}},[v]_{A_{1}}\right)$.

\section{The $A_{p}$ case}

Proof of Theorem 1.13. Without loss of generality we may assume that $f$ is nonnegative and bounded with compact support. Let $v \in A_{p}$ then $v \in A_{r}, r>p$ with $[v]_{A_{r}} \leq[v]_{A_{p}}$. Fix $t>0$ and let $r>p$ be a parameter that will be chosen in a moment. Since $v \in A_{r}$, in particular, $v d x$ is a doubling weight. Therefore, we can form the Calderón-Zygmund decomposition of $f$ at height $t$ with respect to the measure $v(x) d x$. This yields a collection of disjoint dyadic maximal cubes $\left\{Q_{j}\right\}$, such that for all $Q_{j}$ :

$$
t<\frac{1}{v\left(Q_{j}\right)} \int_{Q_{j}} f(x) v(x) d x \leq \frac{v\left(Q_{j}^{\prime}\right)}{v\left(Q_{j}\right) v\left(Q_{j}^{\prime}\right)} \int_{Q_{j}^{\prime}} f(x) v(x) d x \leq 2^{n r}[v]_{A_{r}} t
$$

where $Q_{j}^{\prime}$ is the ancestor of $Q_{j}$ and where the last inequality is obtained by using standard properties of the $A_{p}$ weights (see Proposition 9.1.5 in $[\mathrm{G}]$ ) and by the maximality property of the $Q_{j}$.

Further, if we let $\Omega:=\cup_{j} Q_{j}$, then $f(x) \leq t$ for almost every $x \in \mathbb{R}^{n} \backslash \Omega$. We decompose $f$ as $g+b$, where

$$
g(x)= \begin{cases}\frac{1}{v\left(Q_{j}\right)} \int_{Q_{j}} f(x) v(x) d x & \text { if } x \in Q_{j} \\ f(x) & \text { if } x \in \mathbb{R}^{n} \backslash \Omega\end{cases}
$$

and let $b(x)=\sum_{j} b_{j}(x)$, with

$$
b_{j}(x)=\left(f(x)-\frac{1}{v\left(Q_{j}\right)} \int_{Q_{j}} f(x) v(x) d x\right) \chi_{Q_{j}}(x) .
$$

If we used this definitions, we have that $g(x) \leq 2^{n r}[v]_{A_{r}} t$ for almost every $x \in \mathbb{R}^{n}$ and

$$
\int_{Q_{j}} b_{j}(x) v(x) d x=0
$$

Following [C-UMP1] if $Q$ is a dyadic cube, then $\forall x \in Q$,

$$
\frac{1}{|Q|} \int_{Q} f(x) v(x) d x=\frac{1}{|Q|} \int_{Q} g(x) v(x) d x+\frac{1}{|Q|} \int_{Q} b(x) v(x) d x \leq M_{d}(g v)(x)+\widetilde{M}_{d}(b v)(x)
$$

where

$$
\widetilde{M}_{d}(h)(x)=\sup _{x \in Q}\left|\frac{1}{|Q|} \int_{Q} h(y) d y\right|
$$


Then, if the supremum is taken over all dyadic cubes containing $x$, we have

$$
M_{d}(f v) \leq M_{d}(g v)+\widetilde{M}_{d}(b v)
$$

Now,

$$
\begin{gathered}
v\left(\left\{x \in \mathbb{R}^{n}: \frac{M_{d}(f v)(x)}{v(x)}>t\right\}\right) \leq v\left(\left\{x \in \mathbb{R}^{n}: \frac{M_{d}(g v)(x)}{v(x)}>t / 2\right\}\right)+ \\
+v\left(\left\{x \in \Omega: \frac{\widetilde{M}_{d}(b v)(x)}{v(x)}>t / 2\right\}\right)+v\left(\left\{x \in \mathbb{R}^{n} \backslash \Omega: \frac{\widetilde{M}_{d}(b v)(x)}{v(x)}>t / 2\right\}\right)=I_{1}+I_{2}+I_{3} .
\end{gathered}
$$

To estimate $I_{1}$ we will use the following improvement of Buckley's theorem (see [Bu]) whose proof can be found in [HP],

Lemma 4.1 Let $1<p<\infty$ and $v \in A_{p}$ then,

$$
\|M\|_{L^{p}(v)} \leq c_{n} p^{\prime}[v]_{A_{p}}^{1 / p}\left[v^{1-p^{\prime}}\right]_{A_{\infty}}^{1 / p}
$$

where $c_{n}$ is a dimensional constant.

We then have after applying Chebyshev inequality

$$
I_{1} \leq \frac{2^{r^{\prime}}}{t^{r^{\prime}}} \int_{\mathbb{R}^{n}} M(g v)^{r^{\prime}} v^{1-r^{\prime}} d x \leq \frac{c_{n}^{r^{\prime}}}{t^{r^{\prime}}} r^{r^{\prime}}[v]_{A_{r}}^{r^{\prime}-1}[v]_{A_{\infty}} \int_{\mathbb{R}^{n}} g^{r^{\prime}} v d x
$$

Since $g(x) \leq 2^{n r}[v]_{A_{r}} t$ and $[v]_{A_{r}} \leq[v]_{A_{p}}$, we have

$$
I_{1} \leq \frac{c_{n}^{r^{\prime}}}{t} r^{r^{\prime}}[v]_{A_{\infty}}[v]_{A_{r}}^{2 r^{\prime}-2} \int_{\mathbb{R}^{n}} g(x) v(x) d x \leq \frac{c_{n}^{r^{\prime}}}{t} r^{r^{\prime}}[v]_{A_{\infty}}[v]_{A_{p}}^{2 r^{\prime}-2} \int_{\mathbb{R}^{n}} g(x) v(x) d x .
$$

Finally, if we let

$$
r=1+\max \left\{p, \log \left(e+[v]_{A_{p}}\right)\right\}
$$

then

$$
r^{\prime}=1+\frac{1}{\max \left\{p, \log \left(e+[v]_{A_{p}}\right)\right\}} .
$$

and a computation shows that $r^{r^{\prime}}$ behaves like $\max \left\{p, \log \left(e+[v]_{A_{p}}\right)\right\}$ and that $[v]_{A_{p}}^{2 r^{\prime}-2}$ is bounded. Therefore,

$$
\begin{aligned}
I_{1} \leq \frac{C_{n}}{t}[v]_{A_{\infty}} \max & \left\{p, \log \left(e+[v]_{A_{p}}\right)\right\}\left(\int_{\mathbb{R}^{n} \backslash \Omega} f(x) v(x) d x+\sum_{j}\left(\frac{1}{v\left(Q_{j}\right)} \int_{Q_{j}} f(x) v(x) d x\right) v\left(Q_{j}\right)\right) \\
\leq & \frac{C_{n}}{t}[v]_{A_{\infty}} \max \left\{p, \log \left(e+[v]_{A_{p}}\right)\right\} \int_{\mathbb{R}^{n}} f(x) v(x) d x .
\end{aligned}
$$

The estimate for $I_{2}$ follows immediately from the properties of the cubes $Q_{j}$ :

$$
I_{2} \leq v(\Omega)=\sum_{j} v\left(Q_{j}\right) \leq \sum_{j} \frac{1}{t} \int_{Q_{j}} f(x) v(x) d x \leq \frac{1}{t} \int_{\mathbb{R}^{n}} f(x) v(x) d x .
$$


Finally, we will prove that $I_{3}=0$. To see this, fix $x \in \mathbb{R}^{n} \backslash \Omega$, since $b$ has support in $\Omega$, to compute $\widetilde{M}_{d}(b v)$ we only need to consider cubes which intersect $\Omega$. Fix such a cube $Q$, and for each $j$ either $Q_{j} \subset Q$ or $Q \cap Q_{j}=\emptyset$. Then, since

$$
\begin{gathered}
\int_{Q_{j}} b_{j}(x) v(x) d x=0 \\
\frac{1}{|Q|} \int_{Q} b(x) v(x) d x=\frac{1}{|Q|} \sum_{j} \int_{Q \cap Q_{j}} b_{j}(x) v(x) d x=\frac{1}{|Q|} \sum_{Q_{j} \subset Q} \int_{Q_{j}} b_{j}(x) v(x) d x=0 .
\end{gathered}
$$

We will use the following lemma for mixed $A_{p}-A_{\infty}$ constants as defined in (16).

Lemma 4.2 Let $p>1$ and let $v \in A_{p}$, then

$$
[v]_{\left(A_{p}\right)^{1 / p}\left(A_{\infty}^{e x p}\right)^{1 / p^{\prime}}} \leq[v]_{A_{p}} \leq[v]_{\left(A_{p}\right)^{1 / p}\left(A_{\infty}^{e x p}\right)^{1 / p^{\prime}}}^{p}
$$

Proof.

The second inequality follows from a simple consequence of a Jensen inequality:

$$
e^{\frac{1}{|Q|} \int_{Q} \log w(x) d x} \leq \frac{1}{|Q|} \int_{Q} w(x) d x
$$

which implies $\left[\left(\frac{1}{|Q|} \int_{Q} w(x) d x\right) e^{\frac{1}{|Q|} \int_{Q} \log w^{-1}(x) d x}\right]^{p-1} \geq 1$ and then

$$
\begin{gathered}
\left(\frac{1}{|Q|} \int_{Q} w(x) d x\right)\left(\frac{1}{|Q|} \int_{Q} w(x)^{1-p^{\prime}} d x\right)^{p-1} \leq \\
\leq\left(\frac{1}{|Q|} \int_{Q} w(x) d x\right)^{p}\left(\frac{1}{|Q|} \int_{Q} w(x)^{1-p^{\prime}} d x\right)^{p-1}\left(e^{\frac{1}{|Q|} \int_{Q} \log w^{-1}(x) d x}\right)^{p-1},
\end{gathered}
$$

whence we obtain

$$
[v]_{A_{p}} \leq[v]_{\left(A_{p}\right)^{1 / p}\left(A_{\infty}^{e x p}\right)^{1 / p^{\prime}}}^{p}
$$

The first inequality also follows from Jensen's inequality in the form

$$
e^{\frac{1}{|Q|} \int_{Q} \log w(x)^{-1} d x} \leq\left(\frac{1}{|Q|} \int_{Q} w(x)^{-\alpha} d x\right)^{1 / \alpha} \quad \alpha>0
$$

considering the case $\alpha=p^{\prime}-1$.

We also need the following lemma that will play an important role in the proof of Theorem 1.15. It is an improvement of Buckley's theorem (see [Bu]) and the proof can be found in $[\mathrm{HP}]$. 
Lemma 4.3 Let $1<p<\infty$ and $v \in A_{p}$ then,

$$
\|M\|_{L^{p}(v)} \leq c_{n} p^{\prime}\left[v^{1-p^{\prime}}\right]_{\left(A_{p^{\prime}}\right)^{1 / p^{\prime}}\left(A_{\infty}^{e x p}\right)^{1 / p}}
$$

where $c_{n}$ is a dimensional constant.

Proof of Theorem 1.15. The structure of the proof is the same as that of Theorem 1.13. The only difference is in the analysis of $I_{1}$. Indeed, combining Chebyshev inequality with Lemma 4.3 we arrive to

$$
I_{1} \leq \frac{2^{r^{\prime}}}{t^{r^{\prime}}} \int_{\mathbb{R}^{n}} M(g v)^{r^{\prime}} v^{1-r^{\prime}} d x \leq \frac{2^{r^{\prime}}}{t^{r^{\prime}}} r^{r^{\prime}}[v]_{\left(A_{r}\right)^{1 / r}\left(A_{\infty}^{e x p}\right)^{1 / r^{\prime}}}^{r^{\prime}} \int_{\mathbb{R}^{n}} g^{r^{\prime}} v d x
$$

and since $g(x) \leq 2^{n r}[v]_{A_{r}} t$ we have

$$
I_{1} \leq \frac{2^{r^{\prime}(1+n)}}{t} r^{r^{\prime}}[v]_{A_{r}}^{r^{\prime}-1}[v]_{\left(A_{r}\right)^{1 / r}\left(A_{\infty}^{e x p}\right)^{1 / r^{\prime}}}^{r^{\prime}} \int_{\mathbb{R}^{n}} g(x) v(x) d x .
$$

As $r>p,[v]_{A_{r}} \leq[v]_{A_{p}}$ and by $(17)[v]_{\left(A_{r}\right)^{1 / r}\left(A_{\infty}^{e x p}\right)^{1 / r^{\prime}}} \leq[v]_{\left(A_{p}\right)^{1 / p}\left(A_{\infty}^{e x p}\right)^{1 / p^{\prime}}}$. Finally, if we let

$$
r=1+\max \left\{p, \log \left(e+[v]_{A_{p}}\right)\right\},
$$

then

$$
r^{\prime}=1+\frac{1}{\max \left\{p, \log \left(e+[v]_{A_{p}}\right)\right\}} .
$$

It is easy to see that $r^{r^{\prime}}$ behaves like

$$
\max \left\{p, \log \left(e+[v]_{A_{p}}\right)\right\}
$$

that $[v]_{\left(A_{p}\right)^{1 / p}\left(A_{\infty}^{e x p}\right)^{1 / p^{\prime}}}^{r^{\prime}}$ behaves like $[v]_{\left(A_{p}\right)^{1 / p}\left(A_{\infty}^{e x p}\right)^{1 / p^{\prime}}}$ and that $[v]_{A_{p}}^{r^{\prime}-1}$ is bounded by a universal constant. Moreover, since $2^{r^{\prime}(1+n)} \leq 2^{2(1+n)}$ we have that

$$
\begin{gathered}
I_{1} \leq \frac{C_{n}}{t}[v]_{\left(A_{p}\right)^{1 / p}\left(A_{\infty}^{e x p}\right)^{1 / p^{\prime}}} \max \left\{p, \log \left(e+[v]_{A_{p}}\right)\right\} \times \\
\times\left(\int_{\mathbb{R}^{n} \backslash \Omega} f(x) v(x) d x+\sum_{j}\left(\frac{1}{v\left(Q_{j}\right)} \int_{Q_{j}} f(x) v(x) d x\right) v\left(Q_{j}\right)\right) .
\end{gathered}
$$

Now by Lemma 4.2 we have

$\max \left\{p, \log \left(e+[v]_{A_{p}}\right)\right\} \leq \max \left\{p, p \log \left(e+[v]_{\left(A_{p}\right)^{1 / p}\left(A_{\infty}^{e x p}\right)^{1 / p^{\prime}}}\right)\right\}=p \log \left(e+[v]_{\left(A_{p}\right)^{1 / p}\left(A_{\infty}^{e x p}\right)^{1 / p^{\prime}}}\right)$ and then

$$
I_{1} \leq \frac{C_{n}}{t} p[v]_{\left(A_{p}\right)^{1 / p}\left(A_{\infty}^{e x p}\right)^{1 / p^{\prime}}} \log \left(e+[v]_{\left(A_{p}\right)^{1 / p}\left(A_{\infty}^{e x p}\right)^{1 / p^{\prime}}}\right) \int_{\mathbb{R}^{n}} f(x) v(x) d x .
$$

This concludes the proof of the theorem. 


\section{Counterexamples}

In this section we show that inequality (13) is false. To do this we proceed by contradiction assuming that this inequality holds. We begin with the following duality argument for any weight $w$,

$$
\|T f\|_{L^{p}(w)}=\sup _{h:\|h\|_{L^{p^{\prime}(w)}}}\left|\int_{\mathbb{R}^{n}} T f h w d x\right| .
$$

Fixing one of these $h$ we have

$$
\int_{\mathbb{R}^{n}} T f h w d x=\int_{\mathbb{R}^{n}} f T^{t}(h w) d x=\int_{\mathbb{R}^{n}} f \frac{T^{t}(h w)}{M w} M w d x
$$

and then

$$
\left|\int_{\mathbb{R}^{n}} T f h w d x\right| \leq\|f\|_{L^{p}(M w)}\left\|\frac{T^{t}(h w)}{M w}\right\|_{L^{p^{\prime}(M w)}}=\|f\|_{L^{p}(M w)}\left\|T^{t} f\right\|_{\left.L^{p^{\prime}}(M w)^{1-p^{\prime}}\right)} .
$$

Now we will use the following lemma which is a particular version of the classical estimate of Coifman-Fefferman for any Calderón-Zygmund operator $T$ : let $p \in(0, \infty)$ and let $w \in A_{\infty}$, then there is a constant $c$ depending upon $p, T$ the $A_{\infty}$ constant of $w$ such that

$$
\|T f\|_{L^{p}(w)} \leq c_{T, p,[w]_{A_{\infty}}}\|M f\|_{L^{p}(w)} .
$$

Then as a consequence we have the special situation: Let $w$ be any weight and let $p \in(1, \infty)$. Then, there is a constant depending only on $p$ and $T$ such that:

$$
\|T f\|_{\left.L^{p}(M w)^{1-p}\right)} \leq c\|M f\|_{\left.L^{p}(M w)^{1-p}\right)} .
$$

This follows from (18) and the fact $(M w)^{1-p} \in A_{\infty}$. Indeed, since $(M w)^{1-p}=(M w)^{\delta(1-2 p)} \in$ $A_{2 p}$ and since $\delta=\frac{p-1}{2 p-1}<\frac{1}{2}$ we have $\left[(M w)^{1-p}\right]_{A_{\infty}} \leq\left[(M w)^{\delta}\right]_{A_{1}}^{2 p-1} \leq c_{n}^{p}$.

It should be mentioned that (19) was improved in [LOP3] and later in [LOP1]. In these papers the relevance was the sharpness of the constant $c$ in term of $p$ which behaves linearly in $p$, but is not important in our context. See also $[R]$ for a similar estimate within the fractional integrals context.

Then, since $T^{t}$ is also a Calderón-Zygmund operator we apply (19)

$\left|\int_{\mathbb{R}^{n}} T f h w d x\right| \leq c_{p, T}\|f\|_{L^{p}(M w)}\|M(h w)\|_{L^{\left.p^{\prime}(M w)^{1-p^{\prime}}\right)}}=c_{p, T}\|f\|_{L^{p}(M w)}\left\|\frac{M(h w)}{M w}\right\|_{L^{p^{\prime}(M w)}}$.

We now apply (13) which is equivalent to

$$
\left\|\frac{M(f w)}{M w}\right\|_{L^{1, \infty}(M w)} \leq c\|f\|_{L^{1}(w)},
$$

and since the operator $f \rightarrow \frac{M(f w)}{M w}$ is trivially bounded on $L^{\infty}$ with constant 1 we apply the Marcinkiewicz's interpolation theorem to deduce

$$
\left|\int_{\mathbb{R}^{n}} T f h w d x\right| \leq c_{p}\|f\|_{L^{p}(M w)}\|h\|_{L^{p^{\prime}(w)}}=c_{p}\|f\|_{L^{p}(M w)} .
$$


Then, for any Calderón-Zygmund operator $T$ and arbitrary weight $w$ we have produced the estimate

$$
\|T f\|_{L^{p}(w)} \leq c_{p}\|f\|_{L^{p}(M w)} .
$$

However, this inequality is well known to be false for any $p \in(1, \infty)$ as was shown by $\mathrm{M}$. Wilson in [W2] for the simplest case, namely the Hilbert transform.

\section{Calderón-Zygmund integral operator}

In this section, we will show the following inequality

$$
\left\|\frac{T(f v)}{v}\right\|_{L^{1, \infty}(v)} \leq C_{n}[v]_{A_{p}} \max \left\{p, \log \left(e+[v]_{A_{p}}\right)\right\} \int_{\mathbb{R}^{n}}|f(x)| v(x) d x .
$$

For the proof of this inequality are need the following two results. The first result was proved in [Hy] and the second result can be found in [GCRdF, p.413].

Theorem 6.1 Let $1<p<\infty, w$ an $A_{p}$-weight and $T$ is a Calderón-Zygmund operator, then

$$
\|T f\|_{L^{p}(w)} \leq c_{n} p p^{\prime}[w]_{A_{p}}^{\max \left(1, \frac{1}{p-1}\right)} .
$$

Lemma 6.2 Let $w$ be a weight. There is a dimensional constant $c_{d}$ such that for all cube $Q$ and for all function $f$ supported in a cube $Q$ with $\int_{Q} f(x) d x=0$, the following inequality is holds:

$$
\int_{\mathbb{R}^{n} \backslash 2 Q}|T f(y)| w(y) d y \leq c_{n} \int_{Q}|f(y)| M w(y) d y .
$$

The structure of the proof of the theorem 1.17 is similar to that of Theorem 1.13.

Proof of Theorem 1.17. Without loss of generality we will assume that $f$ is bounded and has compact support. Since $v \in A_{p}$, then $\forall r>p$, we have $v \in A_{r}$, with $[v]_{A_{r}} \leq[v]_{A_{p}}$. Fix $t>0$. For now let $r>p$ be arbitrary, we will assign a specific value to $r$. Since $v \in A_{r}$, in particular, $v d x$ is a doubling weight. Therefore, we can form the Calderón-Zygmund decomposition of $f$ at height $t$ with respect to the measure $v d x$. This yields a collection of disjoint dyadic maximal cubes $\left\{Q_{j}\right\}$, such that for all $Q_{j}$ :

$$
t<\frac{1}{v\left(Q_{j}\right)} \int_{Q_{j}} f(x) v(x) d x \leq \frac{v\left(Q_{j}^{\prime}\right)}{v\left(Q_{j}\right) v\left(Q_{j}^{\prime}\right)} \int_{Q_{j}^{\prime}} f(x) v(x) d x \leq 2^{n r}[v]_{A_{r}} t
$$

where as before $Q_{j}^{\prime}$ is the ancestor of $Q_{j}$ and where the last inequality is obtained by using standard properties of the $A_{p}$ weights (see Proposition 9.1.5 in $[\mathrm{G}]$ ) and by maximal property of the $Q_{j}$. Further, if we let $\Omega:=\cup_{j} Q_{j}$, then $f(x) \leq t$ for almost every $x \in \mathbb{R}^{n} \backslash \Omega$. We decompose $f$ as $g+b$, where

$$
g(x)= \begin{cases}\frac{1}{v\left(Q_{j}\right)} \int_{Q_{j}} f(x) v(x) d x & \text { if } x \in Q_{j} \\ f(x) & \text { if } x \in \mathbb{R}^{n} \backslash \Omega\end{cases}
$$


and let $b(x)=\sum_{j} b_{j}(x)$, with

$$
b_{j}(x)=\left(f(x)-\frac{1}{v\left(Q_{j}\right)} \int_{Q_{j}} f(x) v(x) d x\right) \chi_{Q_{j}}(x) .
$$

If we used this definitions, we have that $g(x) \leq 2^{n r}[v]_{A_{r}} t$ for almost every $x \in \mathbb{R}^{n}$ and

$$
\int_{Q_{j}} b_{j}(x) v(x) d x=0
$$

Then, since $T$ is a sublineal operator we have that

$$
\begin{gathered}
v\left(\left\{x \in \mathbb{R}^{n}: \frac{|T(f v)(x)|}{v(x)}>t\right\}\right) \leq v\left(\left\{x \in \mathbb{R}^{n}: \frac{|T(g v)(x)|}{v(x)}>t / 2\right\}\right)+ \\
+v\left(\left\{x \in \tilde{\Omega}: \frac{|T(b v)(x)|}{v(x)}>t / 2\right\}\right)+v\left(\left\{x \in \mathbb{R}^{n} \backslash \tilde{\Omega}: \frac{|T(b v)(x)|}{v(x)}>t / 2\right\}\right)=I_{1}+I_{2}+I_{3} .
\end{gathered}
$$

If first we used Chebyshev inequality and later we apply Theorem 6.1 bearing in mind that as $v \in A_{r}$ we have $v^{1-r^{\prime}} \in A_{r^{\prime}}$ with

$$
\left[v^{1-r^{\prime}}\right]_{A_{r^{\prime}}}=[v]_{A_{r}}^{r^{\prime}-1}
$$

Since the exponent of the constant $[v]_{A_{r^{\prime}}}$ in Lemma 6.1 is different if $p>2$ or $p \leq 2$, we have divided the proof into two cases.

Case $p>2$ : In this case, as $r>2$, we have $r^{\prime}<2$ and $\max \left(1, \frac{1}{r^{\prime}-1}\right)=\frac{1}{r^{\prime}-1}$.

$$
I_{1} \leq \frac{2^{r^{\prime}}}{t^{r^{\prime}}} \int_{\mathbb{R}^{n}}|T(g v)(x)|^{r^{\prime}} v(x)^{1-r^{\prime}} d x \leq \frac{c_{n}^{r^{\prime}}}{t^{r^{\prime}}} r^{r^{\prime}}[v]_{A_{r}}^{r^{\prime}} \int_{\mathbb{R}^{n}} g(x)^{r^{\prime}} v(x) d x,
$$

Then, since $g(x) \leq 2^{n r}[v]_{A_{r}} t$ and $[v]_{A_{r}} \leq[v]_{A_{p}}$ we obtened that

$$
I_{1} \leq \frac{c_{n}^{r^{\prime}}}{t} r^{r^{\prime}}[v]_{A_{r}}^{2 r^{\prime}-1} \int_{\mathbb{R}^{n}} g(x) v(x) d x \leq \frac{2^{r^{\prime}(1+n)}}{t} r^{r^{\prime}}[v]_{A_{p}}^{2 r^{\prime}-1} \int_{\mathbb{R}^{n}} g(x) v(x) d x .
$$

As $r>p>2$, we choose

$$
r=1+\max \left\{p, \log \left(e+[v]_{A_{p}}\right)\right\}
$$

then

$$
2>r^{\prime}=1+\frac{1}{\max \left\{p, \log \left(e+[v]_{A_{p}}\right)\right\}} .
$$

For this reason, $r^{r^{\prime}}$ behaves like $\max \left\{p, \log \left(e+[v]_{A_{p}}\right)\right\}$ and $[v]_{A_{p}}^{2 r^{\prime}-1}$ like $[v]_{A_{p}}$.

$$
\begin{gathered}
I_{1} \leq \frac{C_{n}}{t}[v]_{A_{p}} \max \{p \\
\left., \log \left(e+[v]_{A_{p}}\right)\right\}\left(\int_{\mathbb{R}^{n} \backslash \Omega} f(x) v(x) d x+\sum_{j}\left(\frac{1}{v\left(Q_{j}\right)} \int_{Q_{j}} f(x) v(x) d x\right) v\left(Q_{j}\right)\right) \\
\leq \frac{C_{n}}{t}[v]_{A_{p}} \max \left\{p, \log \left(e+[v]_{A_{p}}\right)\right\} \int_{\mathbb{R}^{n}} f(x) v(x) d x .
\end{gathered}
$$


Case $p \leq 2$ : We choose $r=1+2 \log \left(e+[v]_{A_{p}}\right)>2 \geq p$, thus

$$
r^{\prime}=1+\frac{1}{2 \log \left(e+[v]_{A_{p}}\right)}<2
$$

and $\max \left(1, \frac{1}{r^{\prime}-1}\right)=\frac{1}{r^{\prime}-1}$. We can now proceed analogously to the previous case,

$$
I_{1} \leq \frac{c_{d}^{r^{\prime}}}{t} r^{r^{\prime}}[v]_{A_{p}}^{2 r^{\prime}-1} \int_{\mathbb{R}^{n}} g(x) v(x) d x
$$

therefore

$$
I_{1} \leq \frac{c_{d}}{t} \log \left(e+[v]_{A_{p}}\right) \int_{\mathbb{R}^{n}} f(x) v(x) d x .
$$

The estimate for $I_{2}$ follows immediately from the properties of the cubes $Q_{j}$ and from the following inequality

$$
\begin{gathered}
v(2 Q) \leq 2^{n p}[v]_{A_{p}} v(Q) . \\
I_{2} \leq v(\tilde{\Omega}) \leq \sum_{j} v\left(2 Q_{j}\right) \leq 2^{n p}[v]_{A_{p}} \sum_{j} \frac{1}{t} \int_{Q_{j}} f(x) v(x) d x \leq 2^{n p}[v]_{A_{p}} \frac{1}{t} \int_{\mathbb{R}^{n}} f(x) v(x) d x .
\end{gathered}
$$

Finally, to be able to estimate $I_{3}$ we used Lemma 6.2 with $w \equiv 1$.

$$
I_{3} \leq \frac{2}{t} \int_{\mathbb{R}^{n} \backslash \tilde{\Omega}}|T(b v)(x)| d x \leq \frac{2}{t} \sum_{j} \int_{\mathbb{R}^{n} \backslash 2 Q_{j}}\left|T\left(b_{j} v\right)(x)\right| d x \leq \frac{2}{t} \sum_{j} \int_{Q_{j}}\left|b_{j}(x)\right| v(x) d x
$$

if we used $b_{j}$ 's definition, we have that

$$
I_{3} \leq \frac{c_{d}}{t}\|f v\|_{L^{1}\left(\mathbb{R}^{n}\right)}
$$

\section{An adaptation of Sawyer's proof with control of the constant}

In this appendix, we will prove Theorem 1.7 using a method similar as the one considered in [C-UMP1] for proof of theorem 1.4.

The statement of the theorem assumes that the weights belong to the $A_{1}$ class of weights. This weights satisfy a reverse Hölder inequality, namely if $w \in A_{1}$, then there are two constants $r, c>1$ such that

$$
\left(\frac{1}{|Q|} \int_{Q} w^{r}\right)^{1 / r} \leq \frac{c}{|Q|} \int_{Q} w
$$

However, in the classical proofs there is a bad dependence on the constant $c=c\left(r,[w]_{A_{1}}\right)$ and we need a more precise estimate to get our results. 
Lemma 7.1 Let $w \in A_{1}$, and let $r_{w}=1+\frac{1}{2^{n+1}[w] A_{1}}$. Then for any cube $Q$

$$
\left(\frac{1}{|Q|} \int_{Q} w^{r_{w}}\right)^{1 / r_{w}} \leq \frac{2}{|Q|} \int_{Q} w
$$

As a consequence we have that for any cube $Q$ and for any measurable set $E \subset Q$

$$
\frac{w(E)}{w(Q)} \leq 2\left(\frac{|E|}{|I|}\right)^{\epsilon_{w}}
$$

where $\epsilon_{w}=\frac{1}{1+2^{n+1}[w]_{A_{1}}}$.

The proof of this reverse Hölder inequality can be found in [LOP1] and the consequence is an application of Hölder's inequality.

\section{Proof of Theorem 1.7.}

Fix $t>0$ and define $g=f v / t$. Then, it is sufficient to show that

$$
u v\left(\left\{x \in \mathbb{R}^{n}: M_{d}(g)(x)>v(x)\right\}\right) \leq C \int_{\mathbb{R}^{n}}|g(x)| u(x) d x
$$

for any function $g$ bounded with compact support.

Fix $a>2^{n}$. For each $k \in \mathbb{Z}$, let $\left\{I_{j}^{k}\right\}$ be the collection of maximal, disjoint dyadic cubes whose union is the set

$$
\Omega_{k}=\left\{x \in \mathbb{R}^{n}: M_{d} v(x)>a^{k}\right\} \cap\left\{x \in \mathbb{R}^{n}: M_{d} g(x)>a^{k}\right\} .
$$

This decomposition exists since $g$ is bounded and has compact support, so the second set is contained in the union of maximal dyadic cubes. Define

$$
\Gamma=\left\{(k, j):\left|I_{j}^{k} \cap\left\{x: v(x) \leq a^{k+1}\right\}\right|>0\right\} .
$$

As $v \in A_{1}$, we have $M v(x) \leq[v]_{A_{1}} v(x)$ almost everywhere. Hence, for $(k, j) \in \Gamma$

$$
\frac{a^{k}}{[v]_{A_{1}}} \leq \frac{1}{[v]_{A_{1}}} \underset{x \in I_{j}^{k}}{\operatorname{ess} \inf _{d}} M_{d} v(x) \leq \underset{x \in I_{j}^{k}}{\operatorname{ess} \inf } v(x) \leq \frac{1}{\left|I_{j}^{k}\right|} \int_{I_{j}^{k}} v(x) d x \leq[v]_{A_{1}} a^{k+1} .
$$

(Intuitively, if $(k, j) \in \Gamma$, then $I_{j}^{k}$ behaves like a cube from the Calderón-Zygmund decomposition of $v$ at height $a^{k}$ ). Then up to a set of measure zero we have the following inclusions: for each $k$,

$$
\left\{x \in \mathbb{R}^{n}: a^{k}<v(x) \leq a^{k+1}\right\} \cap\left\{x \in \mathbb{R}^{n}: M_{d} g(x)>v(x)\right\} \subset \bigcup_{j:(k, j) \in \Gamma} I_{j}^{k} .
$$

Combining this with (21) we get that

$$
u v\left(\left\{x \in \mathbb{R}^{n}: M_{d} g(x)>v(x)\right\}\right) \leq a[v]_{A_{1}} \sum_{(k, j) \in \Gamma}\left|I_{j}^{k}\right|^{-1} v\left(I_{j}^{k}\right) u\left(I_{j}^{k}\right) .
$$


Fix $N<0$ and define $\Gamma_{N}=\{(k, j) \in \Gamma: k \geq N\}$. We will show that

$$
\sum_{(k, j) \in \gamma_{N}}\left|I_{j}^{k}\right|^{-1} v\left(I_{j}^{k}\right) u\left(I_{j}^{k}\right) \leq C \int_{\mathbb{R}^{n}}|g(x)| u(x) d x .
$$

where the constant $C$ does not depended of $N$. Inequality (20) then follows if we take the limit as $N \rightarrow-\infty$. To prove this, we are going to replace the set of cubes $\left\{I_{j}^{k}\right\}$ by a subset with better properties. First, since $v \in A_{1}$ we can apply Lemma 7.1 and there exists $\epsilon=\left(1+2^{n+1}[v]_{A_{1}}\right)^{-1}>0$ such that given any cube $I$ and $E \subset I$,

$$
\frac{v(E)}{v(I)} \leq 2\left(\frac{|E|}{|I|}\right)^{\epsilon} \text {. }
$$

Fix $\delta$ such that $0<\delta<\epsilon$. Define $\Delta_{N}=\left\{I_{j}^{k}:(k, j) \in \Gamma_{N}\right\}$. The cubes in $\Delta_{N}$ are all dyadic, so they are either paiwise disjoint or ine is contained in the other. For $k>t$, since $\Omega_{k} \subset \Omega_{t}$ and since the cubes $I_{j}^{k}$ are maximal in $\Omega_{k}$, if $I_{s}^{t} \cap I_{j}^{k} \neq \emptyset$, then $I_{j}^{k} \subset I_{s}^{t}$. In particular, each cube $I_{j}^{k} \in \Delta_{N}$ is contained in $\cup_{j} I_{j}^{N} \subset\left\{x: M_{d} g(x)>a^{N}\right\}$. As we noted above, the last set is bounded, so $\Delta_{N}$ contains a maximal disjoint subcollection of cubes.

We form a sequence of sets $\left\{G_{n}\right\}$ by induction. Let $G_{0}$ be the set of all pairs $(k, j) \in \Gamma_{N}$ such that $I_{j}^{k}$ is maximal in $\Delta_{N}$. For $n \geq 0$, given the set $G_{n}$, define the set $G_{n+1}$ to be the set of pairs $(k, j) \in \Gamma_{N}$ such that there exists $(t, s) \in G_{n}$ with $I_{j}^{k} \subsetneq I_{s}^{t}$ and

$$
\begin{aligned}
& \frac{1}{\left|I_{j}^{k}\right|} \int_{I_{j}^{k}} u(x) d x>a^{(k-t) \delta} \frac{1}{\left|I_{s}^{t}\right|} \int_{I_{s}^{t}} u(x) d x, \\
& \frac{1}{\left|I_{i}^{l}\right|} \int_{I_{i}^{l}} u(x) d x \leq a^{(l-t) \delta} \frac{1}{\left|I_{s}^{t}\right|} \int_{I_{s}^{t}} u(x) d x .
\end{aligned}
$$

Whenever $(l, i) \in \Gamma_{N}$ and $I_{j}^{k} \subsetneq I_{i}^{l} \subset I_{s}^{t}$.

Let $P=\cup_{n \geq 0} G_{n}$. Given $(s, t) \in P$, we refer to the cube $I_{s}^{t}$ as a principal cube. Since every cube in $\Delta_{N}$ is contained in a maximal cube, every cube in $\Delta_{N}$ is contained in one or more principal cubes.

To continue, we divide the proof into several steps the same form. We will only look at the behavior of the $A_{1}$-constants and we give the main ideas of the steps.

\section{Step 1}

We claim that

$$
\sum_{(k, j) \in \Gamma_{N}}\left|I_{j}^{k}\right|^{-1} v\left(I_{j}^{k}\right) u\left(I_{j}^{k}\right) \leq C_{\epsilon} \sum_{(k, j) \in P}\left|I_{j}^{k}\right|^{-1} v\left(I_{j}^{k}\right) u\left(I_{j}^{k}\right)
$$

To prove this. Fix $(t, s) \in P$ and let $Q=Q(t, s)$ be the set of indices $(k, j) \in \Gamma_{N}$ such that $I_{j}^{k} \subset I_{s}^{t}$ and $I_{s}^{t}$ is the smallest principal cube containing $I_{j}^{k}$. In particular, each $I_{j}^{k}$ is not a principal cube unless it equals $I_{s}^{t}$.

So by (24) and since $I_{j}^{k} \subset\left\{x: M_{d} v(x)>a^{k}\right\}$,

$$
\sum_{(k, j) \in Q}\left|I_{j}^{k}\right|^{-1} v\left(I_{j}^{k}\right) u\left(I_{j}^{k}\right) \leq\left|I_{s}^{t}\right|^{-1} u\left(I_{s}^{t}\right) \sum_{k \geq t} a^{(k-t) \delta} v\left(I_{s}^{t} \cap\left\{x: M_{d} v(x)>a^{k}\right\}\right) .
$$


By (22), (21), and since $v \in A_{1}$,

$$
v\left(I_{s}^{t} \cap\left\{x: M_{d} v(x)>a^{k}\right\}\right) \leq 2[v]_{A_{1}}^{2 \epsilon} a^{\epsilon} a^{(t-k) \epsilon} v\left(I_{s}^{t}\right) .
$$

Combining these inequalities, we see that

$$
\sum_{(k, j) \in Q}\left|I_{j}^{k}\right|^{-1} v\left(I_{j}^{k}\right) u\left(I_{j}^{k}\right) \leq C_{\epsilon}\left|I_{s}^{t}\right|^{-1} u\left(I_{s}^{t}\right) v\left(I_{s}^{t}\right)
$$

where $C_{\epsilon}=\frac{a^{2 \epsilon-\delta}}{\left(a^{\epsilon-\delta}-1\right)} 2[v]_{A_{1}}^{2 \epsilon}$.

If we now sum over all $(s, t) \in P$, we get $(25)$ since $\cup_{(t, s) \in P} Q(t, s)=\Gamma_{N}$.

Step 2 For each $k$, let $\left\{J_{i}^{k}\right\}$ be the collection of maximal disjoint cubes whose union is $\left\{x: M_{d} g(x)>a^{k}\right\}$. Then

$$
a^{k}<\frac{1}{\left|J_{i}^{k}\right|} \int_{J_{i}^{k}} g(x) d x .
$$

For each $j, I_{j}^{k} \subset\left\{x: M_{d} g(x)>a^{k}\right\}$, so there exists a unique $i=i(j, k)$ such that $I_{j}^{k} \subset J_{i}^{k}$. Hereafter, the index $i$ will always be this function of $(k, j)$. Hence, by (25) and by (21),

$$
\sum_{(k, j) \in \Gamma_{N}}\left|I_{j}^{k}\right|^{-1} v\left(I_{j}^{k}\right) u\left(I_{j}^{k}\right) \leq C_{\epsilon} a[v]_{A_{1}} \int_{\mathbb{R}^{n}} h(x) g(x) d x,
$$

where $h(x)=\sum_{(k, j) \in P}\left|J_{i}^{k}\right|^{-1} \chi_{J_{i}^{k}}(x) u\left(I_{j}^{k}\right)$.

To complete the proof we will show that for each $x, h(x) \leq C u(x)$. Fix $x \in \mathbb{R}^{n}$; without loss of generality we may assume that $u(x)$ is finite. For each $k$, there exists al most one cube $J_{b}^{k}$ such that $x \in J_{b}^{k}$. If it exist, denote this cube by $J^{k}$.

Define $P_{k}=\left\{(k, j) \in P: I_{j}^{k} \subset J^{k}\right\}$, and $G=\left\{k: P_{k} \neq \emptyset\right\}$. We form a sequence $\left\{k_{m}\right\}$ by induction. If $k \in G$, then $k \geq N$, so let $k_{0}$ be the least integer in $G$. Given $K_{m}, m \geq 0$, choose $k_{m+1}>k_{m}$ in $G$ such that

$$
\begin{gathered}
\frac{1}{\mid J^{k_{m+1} \mid}} \int_{J^{k_{m+1}}} u(y) d y>\frac{2}{\left|J^{k_{m}}\right|} \int_{J^{k_{m}}} u(y) d y, \\
\frac{1}{\left|J^{l}\right|} \int_{J^{l}} u(y) d y \leq \frac{2}{\left|J^{k_{m}}\right|} \int_{J^{k_{m}}} u(y) d y, \quad k_{m} \leq l<k_{m+1}, l \in G .
\end{gathered}
$$

Since $u(x)$ is finite, the sequence $\left\{K_{m}\right\}$ only contains a finite number of terms. Then by (27), we have

$$
h(x) \leq \sum_{m} \frac{2}{\left|J^{k_{m}}\right|} \int_{J^{k_{m}}} u(y) d y \sum_{l \in G, k_{m} \leq l<k_{m+1}} \sum_{(l, j) \in P_{l}} \frac{u\left(I_{j}^{l}\right)}{u\left(J^{l}\right)}
$$

we claim that

$$
\sum_{l \in G, k_{m} \leq l<k_{m+1}} \sum_{(l, j) \in P_{l}} \frac{u\left(I_{j}^{l}\right)}{u\left(J^{l}\right)} \leq C_{9}
$$


given this, we would be done: since the sequence $\left\{k_{m}\right\}$ is finite, let $m$ be the largest index. Then by (26) and (28),

$$
h(x) \leq 2 C_{9}\left(2-\left(\frac{1}{2}\right)^{m}\right)[u]_{A_{1}} u(x) .
$$

Therefore, to complete the proof we must show (28). We do this in two steps.

Step 3 They proved in [C-UMP1] that if $(l, j) \in P_{l}, k_{m} \leq l<k_{m+1}$, then

$$
\frac{1}{\left|I_{j}^{l}\right|} \int_{I_{j}^{l}} u(y) d y>\frac{a^{\left(l-k_{m}\right) \delta}}{2[u]_{A_{1}}} \frac{1}{\left|J^{l}\right|} \int_{J^{l}} u(y) d y .
$$

Step 4 We will now (28). By (29) and again since $u \in A_{1}$, if $y \in I_{j}^{l}$, Then

$$
\lambda=\frac{a^{\left(l-k_{m}\right) \delta}}{2[u]_{A_{1}}} \frac{u\left(J^{l}\right)}{\left|J^{l}\right|} \frac{1}{[u]_{A_{1}}}<u(y) ;
$$

hence,

$$
\cup_{j:(l, j) \in P_{l}} I_{j}^{l} \subset\left\{x \in J^{l}: u(x)>\lambda\right\} .
$$

For $l$ fixed the cubes $I_{j}^{l}$ are disjoint. Therefore, since $u \in A_{1}$ there exist $\nu=(1+$ $\left.2^{n+1}[u]_{A_{1}}\right)^{-1}$ such that

$$
\sum_{j:(l, j) \in P_{l}} u\left(I_{j}^{l}\right) \leq 2^{1+\nu} u\left(J^{l}\right)[u]_{A_{1}}^{2 \nu} a^{\left(k_{m}-l\right) \delta \nu}
$$

Therefore, we have that

$$
\sum_{l \in G, k_{m} \leq l<k_{m+1}} \sum_{(l, j) \in P_{l}} \frac{u\left(I_{j}^{l}\right)}{u\left(J^{l}\right)} \leq C_{9},
$$

where $C_{9}=2^{1+\nu}[u]_{A_{1}}^{2 \nu} \frac{a^{\delta \nu}}{a^{\delta \nu}-1}$. Then, the constant $C$ of the theorem 1.7 behaves like

$$
\frac{a^{\epsilon-\delta}}{a^{\epsilon-\delta}-1} 2^{3+\nu} a^{\epsilon+2}[v]_{A_{1}}^{2 \epsilon+2}[u]_{A_{1}}^{2 \nu+1} \frac{a^{\delta \nu}}{a^{\delta \nu}-1}\left(2-\left(\frac{1}{2}\right)^{m}\right) \approx[v]_{A_{1}}^{4}[u]_{A_{1}}^{2}
$$

\section{Acknowledgement}

The second author is supported by the Spanish government grant MTM-2014-53850-P, the first and third authors are supported by Universidad Nacional del Sur and CONICET.

Finally, the authors would like to thank the referee whose suggestions and comments have been very helpful to improve the presentation of the paper. 


\section{References}

[AM] K. Andersen and B. Muckenhoupt Weighted weak type Hardy inequalities with applications to Hilbert transforms and maximal functions, Studia Math. 72 (1982), no. 1, 9-26.

[Bu] S.M. Buckley, Estimates for operator norms on weighted spaces and reverse Jensen inequalities, Trans. Amer. Math. Soc., 340 (1) (1993), 253-272.

[C-UMP1] D. Cruz-Uribe, SFO, J.M. Martell and C. Pérez, Weighted weak-type inequalities and a conjetura of Sawyer, Int. Math. Res. Not. IMRN, 30 (2005), 1849-1871.

[C-UMP2] D. Cruz-Uribe, J.M. Martell and C. Pérez, Weights, Extrapolation and the Theory of Rubio de Francia, Birkhäuser Basel, (2011).

[D] J. Duoandikoetxea, Fourier Analysis, Grad. Studies Math. 29, Amer. Math Soc., Providence, 2000.

[F] N. Fujii, Weighted bounded mean oscillation and singular integrals, Math. Japon, 22 (5) (1977/78), 529-534.

[GCRdF] J. García-Cuerva and J.L. Rubio de Francia, Weighted Norm Inequalities and Related Topics, North Holland Math. Studies 116, North Holland, Amsterdam, (1985).

[G] L.Grafakos, Modern Fourier Analysis, in ÔGraduate texts in mathematicsÕ, 250, Springer, New York, 2nd ed., 2009.

[Hr] S.V.Hruščev, A description of weights satisfying the $A_{\infty}$ condition of Muckenhoupt, Proc. Amer. Math. Soc., 90 (2) (1984), 253-257.

[Hy] T. Hytönen, The sharp weighted bound for general Calderón-Zygmund operators, Ann. of Math. (2), 175 (3), (2012). 1473-1506.

[HP] T. Hytöonen and C. Pérez Sharp weighted bounds involving $A_{\infty}$, Analysis \& PDE 6 (2013), 777-818.

[LM] A. Lerner and K. Moen, Mixed $A_{p}-A_{\infty}$ estimates with one supremum, Studia Mathematica, to appear.

[LOP1] A. K. Lerner, S. Ombrosi and C. Pérez, Sharp $A_{1}$ bounds for Calderón-Zygmund operators and the relationship with a problem of Muckenhoupt and Wheeden, Int. Math. Res. Not. IMRN, 2008, no. 6, Art. ID rnm161, 11 pp. 42B20.

[LOP2] A. Lerner, C. Pérez and S. Ombrosi, Weak type estimates for Singular Integrals related to a dual problem of Muckenhoupt-Wheeden, Journal of Fourier analysis and applications, 15 (2009), 394-403 DOI 10.1007/s00041-008-9032-2.

[LOP3] A. Lerner, S. Ombrosi and C. Pérez, $A_{1}$ bounds for Calderón-Zygmund operators related to a problem of Muckenhoupt and Wheeden, Mathematical Research Letters (2009), 16, $149-156$.

[Mu] B. Muckenhoupt, Weighted norm inequaliteies for the Hardy maximal function, Trans. Amer. Math. Soc., 165 (1972), 207-226. 
[MOS] F. J. Martín-Reyes, P. Ortega Salvador and M. D. Sarrión Gavilán, Boundedness of operators of Hardy type in $\Lambda^{p, q}$ spaces and weighted mixed inequalities for singular integral operators, Proc. Roy. Soc. Edinburgh Sect. A 127 (1997), no. 1, 157-170.

[MW] B. Muckenhoupt and R. Wheeden, Some weighted weak-type inequalities for the HardyLittlewood maximal function and the Hilbert transform, Indiana Math. J. 26 (1977), 801-816.

[R] J. Recchi, Mixed $A_{1}-A_{\infty}$ bounds for fractional integrals, J. Math. Anal. Appl. 403 (2013) 283-296.

[Sa] E. Sawyer, $A$ weighted weak type inequality for the maximal function, Proc. Amer. Math. Soc., 93 (1985), 610-614.

[W1] J.M. Wilson, Weighted inequalities for the dyadic square function without dyadic $A_{\infty}$, Duke Math. J., 55 (1) (1987), 19-50.

[W2] J. M. Wilson, Weighted norm inequalities for the continuos square functions, Trans. Amer. Math. Soc. 314 (1989), 661-692.

[W3] J.M. Wilson, Weigthed Littlewood-Paley theory and exponential-square integrability, Lecture Notes in Math., Springer, Berlin, 1924 (2008).

Sheldy Ombrosi, Departamento de Matemáticas, Universidad Nacional del Sur, 8000 Bahia Blanca, Argentina. e-mail address: sombrosi@uns.edu.ar

Carlos Pérez, Department of Mathematics, University of the Basque Country and Ikerbasque, Bilbao, Spain e-mail address: carlos.perezmo@ehu.es

Jorgelina Recchi, Departamento De Matemáticas, Universidad Nacional Del Sur, 8000 Bahia Blanca, Argentina. e-mail address: drecchi@uns.edu.ar 